\title{
THE BERGMAN KERNEL FUNCTION
}

\author{
GADADHAR MISRA
}

\begin{abstract}
In this note, we point out that a large family of $n \times n$ matrix valued kernel functions defined on the unit disc $\mathbb{D} \subseteq \mathbb{C}$, which were constructed recently in [9], behave like the familiar Bergman kernel function on $\mathbb{D}$ in several different ways. We show that a number of questions involving the multiplication operator on the corresponding Hilbert space of holomorphic functions on $\mathbb{D}$ can be answered using this likeness.
\end{abstract}

\section{INTRODUCTION}

Let $\mathcal{D}$ be a bounded open connected subset of $\mathbb{C}^{m}$ and $\mathbb{A}^{2}(\mathcal{D})$ be the Hilbert space of square integrable (with respect to the volume measure) holomorphic functions on $\mathcal{D}$. The Bergman kernel function (cf. [1]) $B_{\mathcal{D}}: \mathcal{D} \times \mathcal{D} \rightarrow \mathbb{C}$ is uniquely defined by the two requirements:

(i) $\quad B_{\mathcal{D}}(\cdot, w) \in \mathbb{A}^{2}(\mathcal{D})$ for all $w \in \mathcal{D}$, and

(ii) $\left\langle f, B_{\mathcal{D}}(\cdot, w)\right\rangle=f(w)$ for all $f \in \mathbb{A}^{2}(\mathcal{D})$.

Let $e_{n}(z), n \geq 0$, be an orthonormal basis for the Bergman space $\mathbb{A}^{2}(\mathcal{D})$. Any $f \in \mathbb{A}^{2}(\mathcal{D})$ has the Fourier series expansion $f(z)=\sum_{n=0}^{\infty} a_{n} e_{n}(z)$. Assuming that the sum

$$
B(z, w):=\sum_{n=0}^{\infty} e_{n}(z) \overline{e_{n}(w)}
$$

is in $\mathbb{A}^{2}(\mathcal{D})$ for each $w \in \mathcal{D}$, we see that

$$
\langle f(z), B(z, w)\rangle=f(w), w \in \mathcal{D} .
$$

For the Bergman space of the disc $\mathbb{A}^{2}(\mathbb{D})$, clearly the monomials $z^{n}$ are orthogonal with respect to the measure $\frac{1}{\pi} r d r d \theta$ and $\left\|z^{n}\right\|=\frac{1}{\sqrt{n+1}}$. Therefore, $\left\{\sqrt{n+1} z^{n}: n \geq 0\right\}$ forms an orthonormal basis in the Bergman space of the unit disc $\mathbb{D}$. Hence the Bergman kernel $B_{\mathbb{D}}$ of the unit disc is given by the formula:

$$
B_{\mathbb{D}}(z, w)=\sum_{n=0}^{\infty}(n+1) z^{n} \bar{w}^{n}=(1-z \bar{w})^{-2}
$$

Similarly, it is not hard to verify that $\left\{\sqrt{\left(\begin{array}{c}-m-1 \\ |I|\end{array}\right)\left(\begin{array}{c}I \\ |I|\end{array}\right)} z^{I}: I=\left(i_{1}, \ldots, i_{m}\right)\right\}$, where $z^{I}=z_{i}^{i_{1}} \cdots z_{m}^{i_{m}}$ for $z \in \mathbb{B}^{m}$, is an orthonormal basis for the Bergman space $\mathbb{A}^{2}\left(\mathbb{B}^{m}\right)$ of the unit ball $\mathbb{B}^{m}$. It follows, as above, that

$$
B_{\mathbb{B}^{m}}(z, w)=\sum_{|I|=0}^{\infty}\left(\begin{array}{c}
-m-1 \\
\ell
\end{array}\right)\left(\sum_{|I|=\ell}\left(\begin{array}{c}
|I| \\
I
\end{array}\right) z^{I} \bar{w}^{I}\right)=(1-\langle z, w\rangle)^{-m-1}
$$

This work is supported, in part, by the Department of Science and Technology, Government of India. 
The Bergman Kernel $B_{\mathcal{D}}$ transforms naturally with respect to bi-holomorphic transformations $\varphi: \mathcal{D} \rightarrow \mathcal{D}^{\prime}$, that is, if $\varphi: \mathcal{D} \rightarrow \mathcal{D}^{\prime}$ is a holomorphic map then

$$
B_{\mathcal{D}}(\varphi(z), \varphi(w))=J(\varphi, z)^{-1} B_{\mathcal{D}}(z, w) \overline{J(\varphi, w)}^{-1}, z, w \in \mathcal{D}
$$

where $J(\varphi, z)$ is the determinant of the complex Jacobian of the map $\varphi$ at $z$. This striking property makes it quasi-invariant. It follows that the Bergman metric

$$
\sum_{i, j=1}^{m} \frac{\partial^{2}}{\bar{\partial} z_{j} \partial z_{i}} \log B_{\mathcal{D}}(z, z) d z_{i} \wedge d \bar{z}_{j}
$$

is a bi-holomorphic invariant for the domain $\mathcal{D}$.

The quasi-invariance of $B$ is equivalent to saying that the map $U(\varphi): \mathbb{A}^{2}\left(\mathcal{D}^{\prime}\right) \rightarrow \mathbb{A}^{2}(\mathcal{D})$ defined by the formula:

$$
U(\varphi) f)(z)=J\left(\varphi^{-1}, z\right)\left(f \circ \varphi^{-1}\right)(z), f \in \mathbb{A}^{2}\left(\mathcal{D}^{\prime}\right), z \in \mathcal{D}
$$

is an isometry.

The map $J: \operatorname{Aut}(\mathcal{D}) \times \mathcal{D} \rightarrow \mathbb{C}$ satisfies the cocycle property, namely,

$$
J(\psi \varphi, z)=J(\varphi, \psi(z)) J(\psi, z), \varphi, \psi \in \operatorname{Aut}(\mathcal{D}), z \in \mathcal{D} .
$$

This ensures that the map $U: \operatorname{Aut}(\mathcal{D}) \rightarrow \mathcal{U}\left(\mathbb{A}^{2}(\mathcal{D})\right)$, where $\mathcal{U}\left(\mathbb{A}^{2}(\mathcal{D})\right)$ is the group of unitary operators on the Bergman space $\mathbb{A}^{2}(\mathcal{D})$, is a homomorphism.

As a second consequence of the quasi invariance, we point out that if the bi-holomorphic automorphism group acts transitively on the domain $\mathcal{D}$, then

$$
B_{\mathcal{D}}(z, z)=J(\varphi, z)^{-1} B_{\mathcal{D}}(0,0) \overline{J(\varphi, 0)}^{-1},
$$

where $z=\varphi(0)$ for some $\varphi \in \operatorname{Aut}(\mathcal{D})$. Thus the Bergman kernel $B_{\mathcal{D}}$ is explicitly determined from the computation of $B_{\mathcal{D}}(0,0)$ and the Jacobian $J(\varphi, 0), \varphi \in \operatorname{Aut}(\mathcal{D})$.

Often, the automorphism group $\operatorname{Aut}(\mathcal{D})$ has additional structure. For instance, if $\mathcal{D}$ is taken to be a bounded symmetric domain, then $\operatorname{Aut}(\mathcal{D})$ is a Lie group and acts transitively on $\mathcal{D}$. In this case, the group $\operatorname{Aut}(\mathcal{D})$ is a topological group. The group of unitary operators on any separable complex Hilbert space, in particular the Bergman space, inherits the strong topology from the space of all bounded operators on it. The map $U: \operatorname{Aut}(\mathcal{D}) \rightarrow \mathcal{U}\left(\mathbb{A}^{2}(\mathcal{D})\right)$, described above, is continuous. Such a map, a continuous unitary homomorphism, is called a unitary representation. In the following, we will assume that $\mathcal{D}$ is a bounded symmetric domain in $\mathbb{C}^{m}$.

\section{Berezin-Wallach Set}

One easily exploits the quasi-invariance of the Bergman kernel to construct new unitary representations of the automorphism group $\operatorname{Aut}(\mathcal{D})$. Assuming that $B(z, w)$ is non-zero and $\mathcal{D}$ is simply connected, we define $B^{(\lambda)}(z, w):=(B(z, w))^{\lambda}, z, w \in \mathcal{D}, \lambda>0$, taking a continuous branch of the logarithm. Now, as before,

$$
B^{(\lambda)}(\varphi(z), \varphi(w))=J(\varphi, z)^{-\lambda} B^{(\lambda)}(z, w) \overline{J(\varphi, w)}^{-\lambda}, \varphi \in \operatorname{Aut}(\mathcal{D}), z, w \in \mathcal{D},
$$

where for a fixed $\varphi \in \operatorname{Aut}(\mathcal{D})$, the function $J(\varphi, z)^{-\lambda}$ is defined on $\mathcal{D}$ using a continuous branch of the logarithm. However, for $\lambda>0$, to ensure continuity of the multiplier $J^{(\lambda)}:=J^{\lambda}: G \times \mathcal{D} \rightarrow \mathbb{C}$, in the first variable, it is necessary to work with the universal covering group $\tilde{G}:=\widetilde{\operatorname{Aut}(\mathcal{D})}$ of the automorphism group $G:=\operatorname{Aut}(\mathcal{D})[4,3,9]$. Let $\pi: \tilde{G} \rightarrow G$ be the quotient map. It then follows 
that $J^{(\lambda)}(\tilde{\varphi}, z):=(J(\pi(\tilde{\varphi}), z))^{\lambda}, \tilde{\varphi} \in \tilde{G}, z \in \mathcal{D}$ is well-defined on $\tilde{G} \times \mathcal{D}$. It satisfies the co-cycle property. Thus the map

$$
U^{(\lambda)}: \tilde{G} \rightarrow \operatorname{End}(\operatorname{Hol}(\mathcal{D}))
$$

given by the formula

$$
\left(U^{(\lambda)}(\varphi) f\right)(z)=J\left(\varphi^{-1}, z\right)^{\lambda}\left(f \circ \varphi^{-1}\right)(z), \varphi \in \tilde{G}, f \in \operatorname{Hol}(\mathcal{D})
$$

is a homomorphism.

It is natural to ask if there exists a Hilbert space $\mathcal{H} \subseteq \operatorname{Hol}(\mathcal{D})$ such that

$$
\left\{U^{(\lambda)}(\tilde{\varphi}): \tilde{\varphi} \in \tilde{G}\right\} \subseteq \mathcal{U}(\mathcal{H}),
$$

where $\mathcal{U}(\mathcal{H})$ is the group of unitary operators on the Hilbert space $\mathcal{H}$. An affirmative answer will ensure the existence of a unitary representation $U^{(\lambda)}$ of the group $\tilde{G}$. Fortunately, there are two different ways in which we can obtain an answer to this question.

Let $Q: \mathcal{D} \rightarrow \mathcal{M}_{n}$ be a real analytic and assume that $Q(z)>0$ for $z \in \mathcal{D}$. For the map $U^{(\lambda)}(\varphi)$, $\varphi \in G$, to be isometric on the Hilbert space

$$
\mathbb{A}^{2}(\mathcal{D}, Q d V)=\left\{f: \mathcal{D} \rightarrow \mathbb{C}^{n}: f \text { holomorphic and } \int_{\mathcal{D}}\langle f(z), Q(z) f(z)\rangle d V(z)<\infty\right\},
$$

we must have

$$
\begin{aligned}
\int_{\mathcal{D}} & \overline{f(\varphi(z))} \overline{J(\varphi, z)}{ }^{\lambda} Q(z) J(\varphi, z)^{\lambda} f(\varphi(z)) d V(z) \\
\quad= & \int_{\mathcal{D}} \overline{f(w)} Q(w) f(w) d V(w) \\
\quad= & \int_{\mathcal{D}} \overline{f(\varphi(z))} Q(\varphi(z)) f(\varphi(z))|J(\varphi, z)|^{2} d V(z), f \in \mathbb{A}^{2}(\mathcal{D}, Q d V)
\end{aligned}
$$

This amounts to the transformation rule

$$
Q(\varphi(z))=\overline{J(\varphi, z)})^{\lambda} Q(z) J(\varphi, z)^{\lambda}|J(\varphi, z)|^{-2}
$$

for the weight function $Q$.

ExAmPle 2.1. In the case of the unit disc $\mathcal{D}=\mathbb{D}$, the automorphism group is transitive, picking a $\varphi:=\varphi_{z}$ such that $\varphi_{z}(0)=z$, we see that $Q(z)=\left(1-|z|^{2}\right)^{2 \lambda-2}$.

However, the Hilbert space

$$
\mathbb{A}^{2}\left(\mathbb{D},\left(1-|z|^{2}\right)^{2 \lambda-2} d V(z)\right)
$$

is non-zero if and only if $2 \lambda-2>-1$. Thus we must have $\lambda>\frac{1}{2}$. The Hardy space with its reproducing kernel $\mathbb{S}(z, w)=(1-z \bar{w})^{-1}$ takes care of the case $\lambda=\frac{1}{2}$. However, if $\lambda<\frac{1}{2}$, then there exists no measure inducing such an inner product. Never the less, since $B^{(\lambda)}=(1-z \bar{w})^{-\frac{\lambda}{2}}$ is a positive definite kernel on the disc $\mathbb{D}$, there is a Hilbert space $\mathbb{A}^{(\lambda)}$ consisting of holomorphic functions defined on the disc $\mathbb{D}$ with the following properties:

(i) $\quad B^{(\lambda)}(\cdot, w) \in \mathbb{A}^{(\lambda)}(\mathbb{D})$ for all $w \in \mathbb{D}$, and

(ii) $\left\langle f, B^{(\lambda)}(\cdot, w)\right\rangle=f(w)$ for all $f \in A^{(\lambda)}(\mathbb{D})$.

These two properties determine $B^{(\lambda)}$ uniquely. The function $B^{(\lambda)}$ is called the reproducing kernel for the Hilbert space $\mathbb{A}^{(\lambda)}(\mathbb{D})$. 
If we assume $Q \geq 0$ is a scalar valued function and that the Hilbert space $\mathbb{A}^{2}(\mathcal{D}, Q d V) \neq\{0\}$, then polarizing $Q$, we see that the corresponding reproducing kernel $B^{(\lambda)}(z, z)$ must be, up to a positive constant, of the form $Q(z, z)^{-1}\left|J\left(\varphi_{z}, z\right)\right|^{2}=\left|J\left(\varphi_{z}, z\right)\right|^{-2 \lambda}$ for some $\lambda>0$. Here, $\varphi_{z}$ is any element of $G$ with $\varphi_{z}(z)=0$. For a bounded symmetric domain $\mathcal{D}$, it can be seen that $\mathbb{A}^{(\lambda)}(\mathcal{D}):=\mathbb{A}^{2}(\mathcal{D}, Q d V)$ is non-zero for some $\lambda>\lambda_{0}$. But what about an arbitrary $\lambda>0$ ? It is possible that $B^{(\lambda)}$ is positive definite for some $0<\lambda \leq \lambda_{0}$. For such a $\lambda$, there is a recipe to construct a Hilbert space $\mathbb{A}^{(\lambda)}(\mathcal{D})$ whose reproducing kernel is $B^{(\lambda)}$. The Hilbert space $\mathbb{A}^{(\lambda)}(\mathcal{D})$ is the completion of the linear span of all vectors in the set $\mathcal{S}=\left\{B^{(\lambda)}(\cdot, w), w \in \mathcal{D}\right\}$, where the inner product between two of the vectors from $\mathcal{S}$ is defined by

$$
\left\langle B^{(\lambda)}(\cdot, u), B^{(\lambda)}(\cdot, w)\right\rangle=B^{(\lambda)}(w, u), \text { for } u, w \in \mathcal{D} .
$$

The positive definiteness of the Kernel $B^{(\lambda)}$ guarantees that the natural extension, of the formula given above, to the linear span $\mathcal{S}^{\circ}$ of the set $\mathcal{S}$ defines an inner product. It is then apparent that $B^{(\lambda)}$ has the reproducing property on $\mathcal{S}^{\circ}$. The Hilbert space $\mathbb{A}^{(\lambda)}(\mathcal{D})$ is the completeion of $\mathcal{S}^{\circ}$.

The Berezin-Wallach set

$$
\mathcal{W}_{\mathcal{D}}:=\left\{\lambda>0: B^{(\lambda)} \text { is positive definite }\right\}
$$

first appeared in the work of Berizin [5,6]. It is clear that $\lambda \in \mathcal{W}_{\mathcal{D}}$ if and only if there is a Hilbert space $\mathbb{A}^{(\lambda)}(\mathcal{D})$ of holomorphic functions on $\mathcal{D}$ whose reproducing kernel is $B^{(\lambda)}$. Then the map $U^{(\lambda)}$ defines a unitary representation of the group $\tilde{G}$. An extensive discussion these representations appears in [12]. An explicit description of the Berezin-Wallach set for bounded symmetric domains is given in [8]. Some of the operator theoretic properties of the commuting tuple of multiplication operators $\left(M_{1}, \ldots, M_{m}\right)$ on $\mathbb{A}^{(\lambda)}(\mathcal{D})$ appears in [2]. Here the multiplication operator $M_{i}: \mathbb{A}^{(\lambda)}(\mathcal{D}) \rightarrow \mathbb{A}^{(\lambda)}(\mathcal{D})$, $1 \leq i \leq m$, is given by the formula $\left(M_{i} f\right)(z)=z_{i} f(z)$ for $f \in \mathbb{A}^{(\lambda)}(\mathcal{D})$ and $z \in \mathcal{D}$.

In the case of the unit ball $\mathbb{B}^{m}$, the Wallach set $\mathcal{W}_{\mathbb{B}^{m}}=\mathbb{R}_{+}$and $\lambda_{0}=\frac{m}{m+1}$. The inner product of the Hilbert spaces $\mathbb{A}^{(\lambda)}$ for $\lambda<\lambda_{0}=\frac{m}{m+1}$ cannot be written as an integral against a measure with support inside the closed unit ball. This is a restatement of the following operator theoretic phenomenon. The commuting tuple of multiplication operators $M^{(\lambda)}=\left(M_{1}^{(\lambda)}, \ldots, M_{1}^{(\lambda)}\right)$ with $M_{i}^{(\lambda)}$ : $\mathbb{A}^{(\lambda)}\left(\mathbb{B}^{m}\right) \rightarrow \mathbb{A}^{(\lambda)}\left(\mathbb{B}^{m}\right)$ is subnormal if and only if $\lambda \geq \lambda_{0}=\frac{m}{m+1}$ as was pointed out in [2].

\section{VECTOR VALUED FUnCTIONS}

What about constructing unitary representations of the group $\tilde{G}$ on some Hilbert space of holomorphic functions on $\mathcal{D}$ taking values in $\mathbb{C}^{n}$. To this end, first, we must answer the following question.

Question 3.1. Does there exist a positive definite kernel function

$$
\mathbf{B}: \mathcal{D} \times \mathcal{D} \rightarrow \mathbb{C}^{n \times n}
$$

possessing the quasi-invariance property of the Bergman kernel function

$$
\mathbf{B}(\varphi(z), \varphi(w))=J(\varphi, z)^{-1} \mathbf{B}(z, w)\left(J(\varphi, w)^{*}\right)^{-1},
$$

for some cocycle $J: \operatorname{Aut}(\mathcal{D}) \times \mathcal{D} \rightarrow \mathbb{C}^{n \times n}$ ? 
If there is a positive definite kernel function $\mathbf{B}$ as above, then the existence of a Hilbert space $\mathcal{H}$ of holomorphic functions on $\mathcal{D}$ taking values in $\mathbb{C}^{n}$ with the property:

$$
\begin{aligned}
\mathbf{B}_{w}(\cdot) \zeta \in \mathcal{H} & \text { for all } w \in \mathcal{D} \text { and } \zeta \in \mathbb{C}^{n} \\
\langle f(w), \zeta\rangle=\left\langle f(\cdot), \mathbf{B}_{w}(\cdot) \zeta\right\rangle & \text { for all } f \in \mathcal{H} \text { and } \zeta \in \mathbb{C}^{n}
\end{aligned}
$$

follows from a slight generalization of the construction we have outlined (cf. [10]). As before, the transformation rule of $\mathbf{B}$ ensures that the natural map

$$
U: \operatorname{Aut}(\mathcal{D}) \rightarrow \operatorname{End}\left(\operatorname{Hol}\left(\mathcal{D}, \mathbb{C}^{n}\right)\right)
$$

given by

$$
(U(\varphi) f)(z)=J\left(\varphi^{-1}, z\right)\left(f \circ \varphi^{-1}\right)(z)
$$

is also unitray, that is, $U(\varphi)$ is unitary on $\mathcal{H}$ for $\varphi$ in $\widetilde{A u t(\mathcal{D}})$. Since $J$ is assumed to be a cocycle, the $\operatorname{map} \varphi \mapsto U(\varphi)$ is a homomorphism of $\tilde{G}$ also. We now investigate the possibility of the existence of the kernel function $\mathbf{B}$, in detail, in the case of $\mathcal{D}=\mathbb{D}$.

3.1. The unit disc. The automorphism group of the unit disc $\mathbb{D}$ is easily described:

$$
\operatorname{Aut}(\mathbb{D})=\left\{\varphi_{\theta, a}: 0 \leq \theta<2 \pi, a \in \mathbb{D}\right\},
$$

where $\varphi_{\theta, a}(z)=e^{i \theta}(z-a)(1-\bar{a} z)^{-1}$. As a topological group Möb is $\mathbb{T} \times \mathbb{D}$. More interesting is the two fold covering group $S U(1,1)$

$$
S U(1,1)=\left\{\left(\begin{array}{cc}
a & b \\
\bar{b} & \bar{a}
\end{array}\right):|a|^{2}-|b|^{2}=1\right\}
$$

which acts on the unit disc $\mathbb{D}$ according to the rule $g \cdot z=(a z+b)(\bar{b} z+\bar{a})^{-1}, g \in S U(1,1)$. For $\lambda>0$,

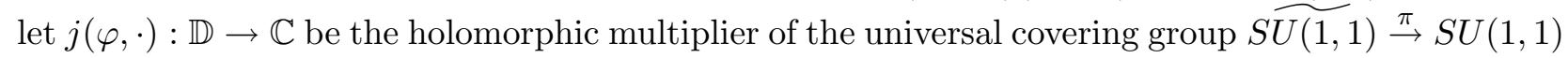
defined by

$$
j(\tilde{\varphi}, z)=\left(\frac{\partial \varphi}{\partial z}(z)\right)^{\lambda}=(\bar{b} z+a)^{-2 \lambda}
$$

for $\tilde{\varphi} \in \widehat{S U(1,1)}$ with $\varphi=\pi(\tilde{\varphi})=\left(\frac{a}{b} \frac{b}{a}\right) \in S U(1,1)$.

Let $\varrho$ be an irreducible representation of $\operatorname{Aut}(\mathbb{D})$ on $\mathbb{C}^{m+1}$. Then

$$
J_{\varrho}(\tilde{\varphi}, z)=\left(\varphi^{\prime}\right)^{\lambda}(z) \varrho(\tilde{\varphi}), \tilde{\varphi} \in \widetilde{\operatorname{Aut}(\mathbb{D})}, \pi(\tilde{\varphi})=\varphi, z \in \mathbb{D}
$$

is a multiplier on the group $\widehat{\operatorname{Aut}(\mathbb{D})}$. Here is an explicit formula for $J_{\varrho}$ :

$$
J_{\varrho}(\tilde{\varphi}, z)=\left(\varphi^{\prime}\right)^{2 \lambda-\frac{m}{2}}(z) D(\varphi)^{\frac{1}{2}} \exp \left(c_{\varphi} S_{m}\right) D(\varphi)^{\frac{1}{2}},
$$

where $S_{m}$ is the forward shift with weights $\{1,2, \ldots, m\}$ and $D(\varphi)$ is a diagonal matrix whose diagonal sequence is $\left\{\left(\varphi^{\prime}\right)^{m}(z),\left(\varphi^{\prime}\right)^{m-1}(z), \ldots, 1\right\}$.

We construct the Hilbert space $\mathcal{H}^{(\lambda, \varrho)}$ of square integrable holomorphic functions on the unit disc with respect to the measure $Q(z) d V(z)$ with

$$
Q(z):=J\left(\varphi_{z}, 0\right)^{*} Q(0) J\left(\varphi_{z}, 0\right)\left|\varphi^{\prime}(z)\right|^{-2},
$$

where $\varphi_{z}(0)=z$. For the Hilbert space $\mathcal{H}^{(\lambda, \varrho)}$ to be non-zero, it is necessary and sufficient that $\lambda>\frac{m+1}{2}$.

Consequently, the reproducing kernel $\mathbf{B}^{(\lambda, \varrho)}$ for the Hilbert space $\mathcal{H}^{(\lambda, \varrho)}$ is obtained by polarizing the identity

$$
\mathbf{B}^{(\lambda, \varrho)}(z, z)=J\left(\varphi_{z}, 0\right)^{-1} \mathbf{B}^{(\lambda, \varrho)}(0,0)\left(J\left(\varphi_{z}, 0\right)^{*}\right)^{-1} .
$$


Or, equivalently,

$$
\mathbf{B}^{(\lambda, \varrho)}(z, w)=(1-z \bar{w})^{-(2 \lambda+m)} D(z, \bar{w}) \exp \left(\bar{w} S_{m}\right) \mathbf{B}^{(\lambda, \varrho)}(0,0) \exp \left(z S_{m}^{*}\right) D(z, \bar{w}),
$$

where $D(z, \bar{w})$ is a diagonal matrix with $\left\{(1-z \bar{w})^{m},(1-z \bar{w})^{m-1}, \ldots, 1\right\}$ on its diagonal.

The possible values for the positive diagonal matrix $\mathbf{B}^{(\lambda, \varrho)}(0,0)$ are completely determined by $Q(0)$. Also, $\mathbf{B}^{(\lambda, \varrho)}$ is a positive definite kernel for each choice of $Q(0)$. It is very easy to enlarge this family of quasi-invariant kernels. One sees that

$$
\mathbf{B}^{(\lambda, \varrho)}=B^{(2 \lambda-m)}+\mu_{1}(Q(0)) B^{(2 \lambda-m+2)}+\cdots+\mu_{m}(Q(0)) B^{(2 \lambda+m)},
$$

where $\mu_{1}(Q(0)), \ldots, \mu_{m}(Q(0))$ are some positive real numbers and $B^{(2 \lambda-m+2 j)}$ is a positive definite matrix which can be computed explicitly:

$$
\left(\begin{array}{cc}
\mathbf{0} & \mathbf{0} \\
\mathbf{0} & \left(\left(\partial^{\ell} \bar{\partial}^{k} B^{2 \lambda-m+2 j}(z, w)\right)\right)_{\ell, k=0}^{m-j}
\end{array}\right) .
$$

However, which positive numbers $\mu_{1}(Q(0)), \ldots, \mu_{m}(Q(0))$ appear in the decomposition of $\mathbf{B}^{(\lambda, \varrho)}$ is not easy to determine.

Now, for any $\lambda: \lambda>\frac{m}{2}$ and an arbitrary $m$-tuple $\mu_{1}, \ldots, \mu_{m}$ of positive numbers, the Hilbert space $\mathcal{H}_{\boldsymbol{\mu}}^{(\lambda, \varrho)}$ corresponding to the $(m+1) \times(m+1)$ matrix valued kernel function

$$
\mathbf{B}_{\boldsymbol{\mu}}^{(\lambda, \varrho)}=B^{(2 \lambda-m)}+\mu_{1} B^{(2 \lambda-m+2)}+\cdots+\mu_{m} B^{(2 \lambda+m)}
$$

is non-trivial. Also, since $\mathbf{B}^{(\lambda, \varrho)}$ is quasi-invariant by construction, it follows that the kernel function $\mathbf{B}_{\mu}^{(\lambda, \varrho)}$ is a quasi-invariant on $\mathbb{D}$ as well.

It is natural to investigate the properties of the multiplication operator $M^{(\lambda, \boldsymbol{\mu})}$ on the Hilbert space $\mathcal{H}_{\mu}^{(\lambda, \varrho)}$. Several properties of these operators have been already established in [9].

TheOREM 1. The multiplication operators $M^{(\lambda, \boldsymbol{\mu})}$ for $\lambda>\frac{m}{2}$ and $\mu_{1}, \ldots, \mu_{m}>0$, are bounded, irreducible and belong to the Cowen-Douglas class $B_{m+1}(\mathbb{D})$. They are mutually unitarily inequivalent.

It is proved however, in [11], that these operators are similar unless $\lambda \neq \lambda^{\prime}$. For $m=1$, it is easy to see that the operator $M^{(\lambda, \boldsymbol{\mu})}$ is a contraction if $\lambda \geq 1$ and $\mu_{1}^{2} \geq \frac{1}{2(2 \lambda-1)(\lambda-1)}$. Also, if the operator $M^{(\lambda, \boldsymbol{\mu})}$ is not contractive, it either fails to be power bounded or it is similar to a contractive operator. This verifies the Halmos' conjecture for homogeneous operators in the Cowen - Douglas class. The arguments given above provide an alternative proof of the homogeneity of these operators.

TheOREM 2. For $\lambda>\frac{m}{2}$ and $\mu_{1}, \ldots, \mu_{m}>0$, the multiplication operator $M^{(\lambda, \mu)}$ is homogeneous.

The choice of arbitrary $\mu_{1}, \ldots, \mu_{m}>0$ has made it possible for us to unitarize a much larger class of representations than what would have been possible if we were to insist on the existence of an integral inner product for our Hilbert space. Although, we note that the natural unitary representations that we construct here are all equivalent unless $\lambda \neq \lambda^{\prime}$. Never the less, the homogeneous holomorphic Hermitian vector bundle corresponding to each one of these representations is irreducible. As we had pointed out earlier, the existence of an integral inner product implies that the operator $M^{(\lambda, \boldsymbol{\mu})}$ is subnormal and isolating these is often very important. Along with A. Koranyi, we have shown, for $m=1$, that the homogeneous operator $M^{(\lambda, \boldsymbol{\mu})}$ is subnormal if and only if $\mu_{1}^{2} \geq \frac{\lambda}{(2 \lambda-1)(\lambda-1)}$. 


\section{REFERENCES}

1. S. Bergman, The kernel function and conformal mapping, Amer Math Soc Survey V, 2nd ed, Providence 1970

2. B. Bagchi and G. Misra, Homogeneous tuples of multiplication operators on twisted Bergman space, J. Funct. Anal. 136 (1996), $171-213$.

3. __ Homogeneous operators and projective representations of the Möbius group: a survey, Proc. Ind. Acad. Sc.(Math. Sci.) 111 (2001), 415 - 437.

4. The homogeneous shifts, J. Funct. Anal. 204 (2003), 293 - 319.

5. F. A. Berezin, Quantization in complex bounded domains, Dokl. Akad. Nauk SSSR, 211 (1973), 1263 - 1266; Soviet Math. Dokl, 14 (1973), 1209 - 1213

6. General concept of quantization, Comm. Math. Phys, 40 (1975), $153-174$

7. M. J. Cowen and R. G. Douglas, Complex geometry and operator theory, Acta Math. 141 (1978), $187-261$.

8. J. Faraut and A. Koranyi, Function spaces and reproducing kernels on bounded symmetric domains, J. Funct. Anal., 88 (1990), $64-89$.

9. A. Korányi and G. Misra, Homogeneous operators on Hilbert spaces of holomorphic functions, J. Func. Anal., 254 (2008), $2419-2436$.

10. __ Multiplicity free homogeneous operators in the Cowen-Douglas class, to appear, Perspectives in Mathematical Sciences, Indian Statistical Institute.

11. S. Shyam Roy, Homogeneity, jet construction and similarity, to apopear, J. Complex Analysis Operator Theory.

12. M. Vergne, H. Rossi, Analytic continuation of the holomorphic discrete series of a semi-simple Lie group, Acta Math, 136 (1976), 1 - 59.

Department of Mathematics, Indian Institute of Science, Bangalore 560012

E-mail address: gm@math.iisc.ernet.in 